\title{
Parasitos zoonóticos em fezes de cães de praças públicas no Município de Porto
}

\section{Velho - Rondônia, Brasil}

\author{
Zoonotic parasites in public square dog fees in the City of Porto Velho - Rondônia, Brazil \\ Parásitos zoonóticos en heces de perros de plazas públicas de la Ciudad de Porto Velho - Rondônia,
} Brasil

\section{Resumo}

Cães são hospedeiros definitivos de algumas enfermidades ocasionadas por parasitos, que por vezes os proprietários sequer sabem da necessidade da vermifugação. A contaminação do solo pelas fezes de cães em praças públicas tem sido alvo de diversas pesquisas, afim de revelar a importância desses locais como áreas propícias à transmissão de parasitos. Neste trabalho objetivou-se avaliar a ocorrência de parasitos zoonóticos em fezes de cães com a técnica de WillisMollay, oriundas de praças públicas das diferentes zonas da cidade de Porto Velho - Rondônia, Brasil, sendo elas Zona Sul, Zona Norte, Zona Leste, Zona Oeste e outra da região Central. Foram, ao todo, coletadas 10 fezes como amostragens das regiões citadas acima que, posteriormente, foram processados e averiguados os resultados obtidos através da técnica de Willis- Mollay. Como produto final de amostras conferidas (50), 75\% demonstraram estar positivas para os ovos de Ancylostoma sp. e 25\% para o Toxocara sp. As praças das Zonas Leste e Sul foram as que apresentaram a maior quantidade de amostras contaminadas. Observou-se também a associação dos parasitos Ancylostoma spp. e Toxocara sp. na mesma amostra, vista em todas as praças, sendo mais frequente (20\%) na praça da Zona Leste.

Palavras-chave: Ancylostoma sp.; Toxocara sp.; Fezes; Praças públicas; Zoonoses.

\begin{abstract}
Dogs are definitive hosts of some diseases caused by parasites, which sometimes the owners do not even know about the need for deworming. Soil contamination by dog feces in public squares has been the subject of several researches, in order to reveal the importance of these places as favorable areas for the transmission of parasites. The objective of this work was to evaluate the occurrence of zoonotic parasites in dog feces using the Willis-Mollay technique, from public squares in different areas of the city of Porto Velho - Rondônia, Brazil, namely the South Zone, North Zone, East Zone , West Zone and another in the Central region. A total of 10 feces were collected as a sampling of each region mentioned above, which were later processed and the results obtained through the Willis-Molay technique were investigated. As a final product of checked samples (50), 75\% proved to be positive for Ancylostoma sp. and 25\% for Toxocara sp. The squares in the East and South Zones were the ones with the largest amount of contaminated samples. The association of the parasites
\end{abstract}


Ancylostoma spp. and Toxocara sp. in the same sample, seen in all squares, being more frequent (20\%) in the East Zone square.

Keywords: Ancylostoma sp.; Toxocara sp.; Faeces; Places; Zoonosis.

\section{Resumen}

Los perros son hospedadores definitivos de algunas enfermedades provocadas por parásitos, que en ocasiones los dueños ni siquiera conocen la necesidad de desparasitarse. La contaminación del suelo por heces de perro en plazas públicas ha sido objeto de diversas investigaciones, con el fin de revelar la importancia de estos lugares como áreas favorables para la transmisión de parásitos. El objetivo de este trabajo fue evaluar la ocurrencia de parásitos zoonóticos en heces de perros mediante la técnica Willis-Mollay, de plazas públicas en diferentes áreas de la ciudad de Porto Velho - Rondônia, Brasil, es decir, la Zona Sur, Zona Norte, Zona Este, Zona Oeste y otra en la Región Central. Se recolectaron un total de 10 heces como muestra de cada región mencionada anteriormente, las cuales posteriormente fueron procesadas y se investigaron los resultados obtenidos mediante la técnica Willis-Molay. Como producto final de las muestras controladas (50), el 75\% resultó ser positivo para Ancylostoma sp. y 25\% para Toxocara sp. Las casillas de las Zonas Este y Sur fueron las que presentaron mayor cantidad de muestras contaminadas. La asociación de los parásitos Ancylostoma spp. y Toxocara sp. en la misma muestra, visto en todos los cuadrados, siendo más frecuente (20\%) en el cuadrado de la Zona Este.

Palabras clave: Ancylostoma sp.; Toxocara sp.; Heces; Plazas públicas; Zoonosis.

\section{Introdução}

Um relevante papel dentro da sociedade tem sido manifestado pela presença de animais de companhia, em particular os cães, em proporcionarem não só a companhia, como também estarem colaborando para o amadurecimento físico, emocional e social sobretudo de crianças e pessoas da terceira idade (Blazius, et al., 2005; Raina, et al., 1999). Certos parasitos de potencial zoonótico hospedam os cães de forma definitiva, sendo desconhecida pelos proprietários a necessidade de estarem realizando a everminação para o controle sistemático das endoparasitoses, sendo também a utilização medicamentosa de forma indiscriminada ou até mesmo inadequada, a grande causadora de problemas de resistência, perpetuando assim o ciclo parasitário (Marques, et al., 2012).

A Larva migrans visceral (LMV) destaca-se como um importante agente zoonótico para a saúde pública, causando uma síndrome em humanos, resultante da migração das larvas do Toxocara spp., um ascarídeo com distribuição cosmopolita (Queiroz \& Chiefffi, 2005; Arighi, et al. 2018). A migrans cutânea (LMC é outra zoonose parasitária importante, ocasionada pelos vermes do gênero Ancylostoma. Acomentendo também principalmente as crianças, através do contato com areia e terra, ficando susceptíveis a ter contato imediato com larvas que serão infectantes de nematóides, levando a LMV ou LMC (Lima, 1984; Lima, 2005; Félix et al. 2020).

Os estudos que visam avaliar a contaminação do solo pelas fezes de cães em áreas como as praias e praças públicas tem sido pontos de pesquisas, atestando a sua importância como áreas propícias à transmissão de possíveis agentes parasitários (Capuano \& Rocha, 2006; Gonçalves \& Paludo, 2018; Batista et al., 2019). Com isso, o objetivo deste trabalho foi de avaliar a ocorrência de ovos de parasitos zoonóticos nas fezes de caninos, oriundas de praças públicas na cidade de Porto Velho em Rondônia, Brasil.

\section{Material e Métodos}

O estudo transcorreu entre o período de agosto e outubro do ano de 2020, em cinco praças públicas do município de Porto Velho Rondônia. As praças escolhidas foram uma principal de cada uma das diferentes zonas da cidade, sendo uma da Zona Sul, Zona Leste, Zona Oeste, Zona Norte e outra da região Central.

Para cada praça, foram coletadas uma totalidade de 10 amostras de fezes, sendo elas frescas, conservadas em coletores de fezes que estavam estéreis e, mais tarde, acomodadas em uma caixa térmica que estava com gelo, até a chegada ao laboratório para a realização do processamento. Para a realização da pesquisa dos ovos, foi executada a técnica de Willis-Mollay (1921) 
com utilização de solução salina hipersaturada. Se teve como método de avaliação das amostras a presença ou ausência dos ovos dos agentes parasitários Toxocara spp e Ancylostoma spp. A procura de ovos de parasitos foi efetuatada através de microscopia óptica binocular (CX21 Olympus $\left.{ }^{\circledR}\right)$, empregando as objetivas secas de tamanhos 4x, 10x e 40x.

\section{Resultados e Discussão}

Da totalidade de 50 amostras adquiridas, foi observado que 80\% (40 amostras) delas estavam infestadas com ovos de alguma das espécies dos vermes que estavam sendo pesquisados. Em relação aos parasitos, as amostras positivas demonstraram ovos da espécie Toxocara sp. (25,00\%) e de Ancylostoma spp. (75,00\%), presentes em todas as praças do município, sendo que as praças da zona Leste e Zona Sul, foram as que apresentaram a maior quantidade com amostras infestadas, ambas com $90 \%$ das amostras positivas (Tabela 1).

Tabela 1. Parasitos achados nas amostras de fezes de cães em praças públicas do município de porto Velho em Rondônia, Brasil.

\begin{tabular}{|c|c|c|c|}
\hline Zonas da Cidade & Ancylostoma sp. & Toxocara spp. & Negativas \\
\hline Centro & $60 \%(6 / 10)$ & $10 \%(1 / 10)$ & $30 \%(3 / 10)$ \\
\hline Leste & $80 \%(8 / 10)$ & $40 \%(4 / 10)$ & $10 \%(1 / 10)$ \\
\hline Norte & $60 \%(6 / 10)$ & $0 \%(0 / 10)$ & $30 \%(3 / 10)$ \\
\hline Oeste & $50 \%(5 / 10)$ & $20 \%(2 / 10)$ & $20 \%(2 / 10)$ \\
\hline Sul & $50 \%(5 / 10)$ & $30 \%(3 / 10)$ & $10 \%(1 / 10)$ \\
\hline Total & $60,00 \%(30 / 50)$ & $20,00 \%(10 / 50)$ & $20,00 \%(10 / 50)$ \\
\hline
\end{tabular}

Fonte: Elaborado conforme os resultados de dados coletados nessa pesquisa.

Avaliou-se também a associação dos parasitos Toxocara spp. e Ancylostoma sp. Dentro da mesma amostra, pôde ser observado em todas as praças, sendo de maior frequência (20\%) na praça da Zona Leste (Tabela 2).

Tabela 2. Duplicidade de parasitos encontrados nas amostras de fezes de cães em praças públicas.

\begin{tabular}{lc}
\hline Zonas da Cidade & $\begin{array}{c}\text { Associação entre Toxocara spp. E } \\
\text { Ancylostoma sp. }\end{array}$ \\
\hline Centro & $20 \%(2 / 10)$ \\
Leste & $40 \%(4 / 10)$ \\
Norte & $10 \%(1 / 10)$ \\
Oeste & $20 \%(2 / 10)$ \\
Sul & $20 \%(2 / 10)$ \\
\hline Total & $22,00 \%(11 / 50)$ \\
\hline
\end{tabular}

Fonte: Elaborado conforme os resultados de dados coletados nessa pesquisa.

Na presente pesquisa, é possível se observar uma quantidade expressiva de fezes com contaminação no ambiente de áreas muito frequentadas por cães domiciliados, errantes e peridomiciliados. A percentagem de Ancylostoma sp. (60,00\%) foi mais alta em relação à de Toxocara spp. (20,00\%), os valores de prevalências individuais, como também o geral obtido nesta pesquisa foram bem próximos ao dos estudos de Scaini, et al. (2003), sendo as amostras por eles coletadas em uma praia no município de Cassino; em Cuiabá as amostras foram analisadas por Almeida, et al. (2007); em Belo Horizonte por Ribeiro, et al. (2013); como 
também em Andradina por Coelho, et al. (2011), aos quais respectivamente obtiveram os resultados 71,3\%, 83,3\%, 99\% e $60 \%$ nos seus estudos..

Comumente os cães e gatos são acometidos pelos endoparasitos Ancylostoma sp. $e$ Toxocara spp, com as fezes sendo uma importante forma para a transmissão das zoonoses parasitárias (Santarém, et al., 2004). Os valores referentes aos achados de contaminação por Toxocara spp. está diferente dos achados de Marques et al., (2012) em seu estudo na cidade de Guarulhos sendo de 68,1\%, sendo no estudo dele as menores porcentagens para Ancylostoma sp. O crescente acesso de cães a locais públicos, potencializa o risco de infeção a outros animais, como também para humanos, sendo as crianças por habito geofágicos, principalmente em idade próxima à 5 anos, com grande possibilidade à ingesta de ovos que estão presentes no ambiente (Scaini, et al., 2003; Blazius, 2005). A Organização Mundial da Saúde (OMS), como também diversos autores, defendem a afirmativa da gravidade dessa problemática para a saúde pública (Biasi, et al., 2010). Os autores que fazem essas afirmações, destacam ainda sendo as zoonoses parasitarias, como uma maior problemática em regiões de clima subtropical e tropical, como é o caso da capital Porto Velho, devido as elevadas temperaturas associadas a uma grande umidade, serem as condições perfeitas para os ciclos dos parasitos se completarem. Sendo que os resultados encontrados nessa pesquisa, de grande percentagem de amostras contaminadas, diferindo das regiões frias brasileiras.

As informações geradas nesse trabalho após a análise dos dados são extremamente relevantes, por serem elucidadoras do nível de contaminação por agentes com potencial zoonótico, em locais públicos por contaminação das fezes caninas, colocando assim em risco a suade humana. Auxiliando então na tomada de medidas por médicos e médicos veterinários, como também por outros agentes de saúde envolvidos nesse controle.

Assim faz-se necessário o uso de vermífugos sistematicamente como também o controle e limpeza ambiental. A necessidade de tomada de medidas é importante para a manutenção do processo saúde doença, devido a isso, o médico veterinário tem uma importante função e atuação em diferentes áreas de controle de parasitoses zoonóticas, sendo necessário também uma atenção coletiva as comunidades, embora as medidas de prevenção sejam simples, essas podem ser agravadas por fatores ambientais, por falta de medidas de controle populacional de animais errantes, como também por condições (Campos Filho, et al., 2008; Siqueira \& Fiorini, 1999).

\section{Conclusão}

Este estudo sugere que as praças públicas da cidade de Porto Velho, apresentam valores elevados de contaminação em fezes de cães, por ovos dos parasitos Ancylostoma spp. e Toxocara sp. Apresentando-se como locais de grande potencial para a infecção de outros cães, como também um grande potencial risco à saúde pública

\section{Referências}

Agostini, C., Pegoraro, J., Perpétua, P. C. G., Leonardo, J. M. L. O. (2010). Incidência de Parasitas Intestinais de Caráter Zoonótico em Cães e Gatos na Região de Maringá. V Mostra Interna de Trabalhos de Iniciação Científica. CESUMAR - Centro Universitário de Maringá.

Arighi, P. et al. (2018). Síndrome de larva migrans visceral y abscesso hepático. Reporte de un caso. Arch Argent Pediatr, 116(6): e753-e756 / e753.

Almeida, A. B. P. F. et al. (2007). Contaminação por fezes caninas das praças públicas de Cuiabá, Mato Grosso. Brazilian Journal of Veterinary Research and Animal Science, 44, $132-136$.

Batista, S. P. et al. (2019). Parasitos Zoonóticos em solos de praças públicas no município de Souza, Paraíba. Revista Brasileira de Ciências Veterinárias, 26(3): 82-86.

Biasi, L. A. et al. (2010). Prevalência de enteroparasitoses em crianças de entidade assistencial de Erechim/RS. Revista Perspectiva, Erechim, 34(125), 173 179.

Blazius, R. D., Emerick, S., Prophiro, J. S., Romão, P. R. T., Silva, O. S. (2005). Ocorrência de protozoários e helmintos em amostras de fezes de cães errantes da cidade de Itapema, Santa Catarina. Revista da Sociedade Brasileira de Medicina Tropical, 38(1), 73 - 74. 
Research, Society and Development, v. 10, n. 8, e56110815606, 2021

Bremm, M. (2007). Infecção parasitária por nematódeos em cães do canil municipal de Santa Cruz do Sul/RS. Tese de Especialização, Universidade Federal do Rio Grande do Sul

Campus Filho, P. C. et al. (2008). Parasitas zoonóticos em fezes de cães em praças públicas do município de Itabuna, Bahia, Brasil. Revista Brasileira de Parasitologia Veterinária, 17(4), 206 - 209.

Capuano, D. M. \& Rocha, G. M. (2006). Ocorrência de parasitas com potencial zoonótico em fezes de cães coletadas em áreas públicas do município de Ribeirão Preto, SP, Brasil. Revista Brasileira de Epidemiologia, São Paulo, 9(1), 81-86.

Coelho, L. M. P. S. (2001). Toxocara spp. eggs in public squares of Sorocaba, São Paulo State, Brazil. Revista do Instituto de Medicina Tropical de São Paulo, 43(4), 189-191.

Félix, D. A. S. et al. (2020). Toxocara spp., Larva migrans visceral e saúde pública: Revisão. PUBVET, 14(12): 1-8.

Gonçalves, G. V. \& Paludo, C. A. (2018). Ocorrência de parasitas zoonóticos no solo de praças públicas da cidade de Bento Gonçalves, Rio Grande do Sul. Revista UNINGÁ, 55(2): 72-80.

Lima, W. S. et al. (1984). Surto de larva migrans cutânea em uma creche de Belo Horizonte, MG. Revista do Instituto de Medicina Tropical de São Paulo, 26, $122-124$.

Lima, W. S. (2005). Larva migrans. In: D. V. Neves (Ed. São Paulo: Atheneu), Parasitologia humana, 11 (pp. 271-274).

Marques, J. P. et al. (2012). Contamination of public parks and squares from Guarulhos (São Paulo State, Brazil) by Toxocara spp. and Ancylostoma spp. Revista do Instituto de Medicina Tropical de São Paulo, 54, 267 - 271.

Queiroz, M. L. \& Chieffi, P. P. (2005). Síndrome de Larva migrans visceral e Toxocara migrans. Arq. Med Hosp Fac Cienc Med Santa Casa, 50(3): 117-120.

Raina, P. et al. (1999). Influence of companion animals on the physical and psychological health of older people: an analysis of a one-year longitudinal study. Journal of the American Geriatrics Society, 47, 323 - 329.

Rennó, P. P. et al. (2008). Endoparasitose em aves - revisão de literatura. Revista científica eletrônica de medicina veterinária

Ribeiro, L. M. et al. (2013). Soil contamination in public squares in Belo Horizonte, Minas Gerais, by canine parasites in different developmental stages. Revista do Instituto de Medicina Tropical de São Paulo, 55(4), 229 - 231.

Santarém, V. A., Giufrida, R. \& Zanin, G. A. (2004). Larva Migrans Cutânea: ocorrência de casos humanos e identificação de larvas de Ancylostoma spp em parque público do município de Taciba, São Paulo. Revista da Sociedade Brasileira de Medicina Tropical, 37, 179 - 181.

Scaini, C. J. et al. (2003). Contaminação ambiental por ovos e larvas de helmintos em fezes de cães na área central do Balneário Cassino, Rio Grande do Sul. Revista da Sociedade Brasileira de Medicina Tropical, 36(5), 617 - 619.

Siqueira, R. V. \& Fiorini, J. E. (1999). Conhecimentos e procedimentos de crianças em idade escolar frente a parasitoses intestinais. Revista da Universidade de Alfenas, 5, 215-220.

Souza, L. C. et al. (2001). Associação homem-animal: reflexos na economia. Revista de Educação Continuada CRMV

Vasconcelos, M. G. C. et al. (2008). Isosporose os animais domésticos. Revista científica eletrônica de Medicina Veterinária 